\title{
A Study of the Effect of Relative Permeability and Residual Oil Saturation on Oil Recovery
}

\author{
Mahesh P. Ediriweera $^{1} \quad$ Britt M. Halvorsen ${ }^{1}$ \\ ${ }^{1}$ Process Technology, Telemark University College, Norway
}

\begin{abstract}
Depletion of the production and the low recovery factor are major challenges faced in petroleum industry. The recovery factor and the lifetime of a well are strongly affected by the reservoir properties such as permeability, porosity, residual oil saturation and the water breakthrough time. This research is done to investigate the influence of the relative permeability on oil recovery. Simulations are done for different relative permeability curves and various residual oil saturations. The main focus is the impact on total flow rates and water breakthrough time. The reservoir simulator Rocx in combination with OLGA is used as the simulation tool. The results show that the total oil production and the water breakthrough time are strongly affected by the relative permeability and residual oil saturation. The impact of relative permeability is much higher than the residual oil saturation in petroleum processing.
\end{abstract}

Keywords: oil recovery, OLGA/Rocx, reservoir, properties, relative permeability, water breakthrough, water coning

\section{Introduction}

The offshore petroleum industry is continuously looking for new technologies to enhance the oil recovery and to optimize the operation. The main drawbacks faced by the industry are low oil recovery factor, depletion of oil production, gas coning and water coning. Some previous works have shown that considerable amount of oil still remain in the reservoir after well shutdown. The residual oil saturation and early breakthrough are the main reasons for remaining oil volumes in abandon oil fields. Since the reservoir properties highly influence the oil recovery process, it is interesting to identify the relation between the recovery factor and the reservoir properties. Further, the relative permeability varies with time due to changing in water saturation during the recovery process. If relationships and variations are known, then recovery can be efficiently upgraded by manipulating reservoir properties. Enhanced Oil Recovery (EOR) methods can be used to change the properties such as relative permeability and residual oil saturation. A typical oil reservoir consists of an underlying aquifer and a gas cap. Here in this paper, heavy oil reservoirs are considered and gas coning is not discussed. Heavy oil reservoirs occupy more than two third of global oil reserves.

The oil recovery factor is the ratio between the amount of oil that can be extracted from a reservoir throughout the lifespan of the well and the total reservoir oil in place. The recovery factor is in general less than 20 percent when heavy oil is produced without any external force (P. Zitha, 2011).The conical water profile of the reservoir, which forms above the aquifer during the extraction process, is known as water cone. The water coning phenomena occurs in oil wells with aquifer as shown in Figure 1. The cone spreads towards the well while the amount of oil is being reduced in the reservoir over the life span of the well. When the water cone hits the wellbore, water mixes with oil and the inflow to the well becomes multiphase flow. After the breakthrough, the water volume ratio of the multiphase flow is increased gradually and the volume percentage of the mixture is introduced as water cut.

The demand of oil and gas would remain until a cost effective feasible energy source is found. Hence, the industry always look for new methods to extract as much oil and gas as possible in an efficient and effective way. Recovery mechanisms have been developing for years in order to extract maximum output by increasing the recovery factor and controlling the water breakthrough. These two features are strong functions of reservoir properties and extraction techniques. The regulated reservoir properties expose the access to control the recovery factor and the coning effect. Therefore, an overall understanding over reservoir properties and extraction techniques is desired before going deeper into the improved oil extraction. Tertiary recovery methods, so called Enhance Oil Recovery, which increase the mobility of residual oil throughout the entire reservoir by changing reservoir properties (P. Zitha, 2011). External force or fluids are used to efficiently capture hydrocarbons using these methods. Relative permeability is one of the reservoir properties which can be controlled with existing techniques in the petroleum industry under tertiary recovery methods. 
The purpose of the study is to build an OLGARocx $^{1}$ model for a typical Norwegian oil production process to investigate the influence of relative permeability throughout the operation. Various reservoir models can be used to simulate the characteristics of the reservoir over the period. The model is able to predict the behavior of inflow and the property changes in the reservoir during the normal operation. Only horizontal wells are modelled in this project due to the fact that horizontal wells are the most common technology implemented in Norwegian offshore fields. The TECPLOT ${ }^{2}$ module is used as the result visualization tool to plot the simulated reservoir results.

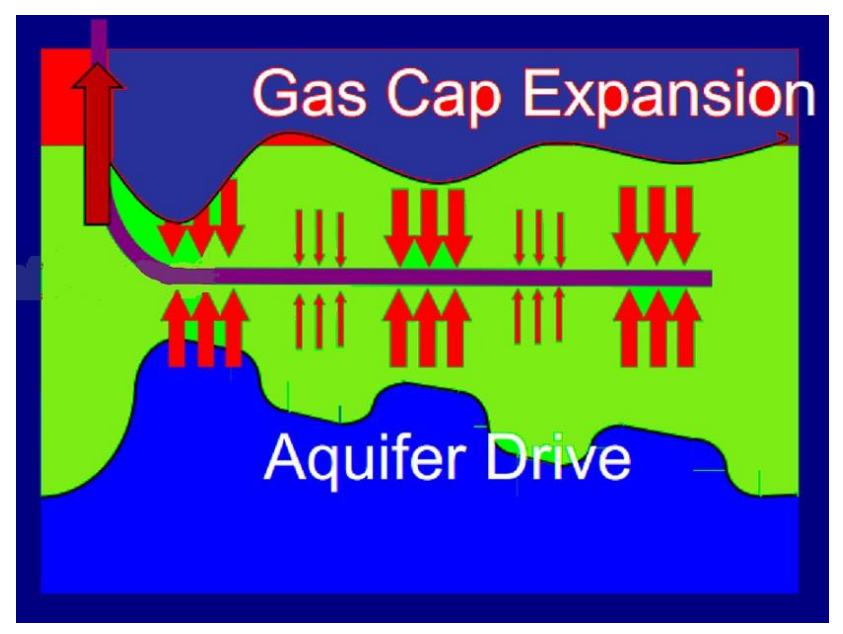

Figure 1. A typical petroleum reservoir with water aquifer and gas cap.

\section{Reservoir Properties}

Each and every petroleum reservoir has its own characteristics throughout the reservoir volume. These properties are distinguished as rock properties and fluid properties. Porosity, permeability and relative permeability are the most critical rock properties. Pressure, temperature, viscosity, specific gravity, oil and gas concentrations are some key fluid properties for the analysis. Conventionally, crude oil is brownish green or black in color with the specific gravity 0.801 0.985 . The distillation process decomposes crude oil into its subcomponents within the boiling point range from $20^{\circ} \mathrm{C}$ to $350^{\circ} \mathrm{C}$ (Speight e Speight, 2009).

\subsection{Porosity and Permeability}

In general, the reservoir volumes consist of sandstone which is considered as high porous and high permeable media. Porosity is a measurement of the void spaces in a rock. Relationship for porosity is expressed as the volume fraction given in Equation 1. Tiny spaces in sandstones hold hydrocarbons and water within the reservoir. Therefore, indirectly the porosity is a dimension of reserved petroleum quantity of the given reservoir. Porosity can be categorized as primary and secondary porosities. Gaps and spaces developed during the sedimentation process is called the primary porosity while the secondary porosity is formed in later stages as dissolving of minerals occurred. Hydrocarbons can only be produced if interconnected pores are available within the reservoir rock. The ratio between interconnected pores and total rock volume is equal to the effective porosity which is the useful property in the oil extraction process. Practically, the porosity value is given in Equation 1 is always higher than the effective porosity.

$$
\begin{aligned}
& \text { Porosity }(\phi) \\
& =\frac{\text { Volume of the Void space }\left(V_{V}\right)}{\text { Total or Bulk Volume of the Rock }\left(V_{T}\right)}
\end{aligned}
$$

Permeability describes the fluid conductivity through a porous media, also known as "absolute/intrinsic permeability". If high pressure is needed to extract hydrocarbons, it is called low permeable reservoirs and vice-versa. Permeability is directly related to the porosity, it depends on the porous connectivity and the size of the porous volumes. Absolute permeability could be determined by laboratory experiments with the use of inert gases (frequently used nitrogen) (A. B. Zolotukhin, 1997). Permeability can be calculated by Darcy's law which was developed semi empirically by Darcy in the $19^{\text {th }}$ century for single phase flow and in $20^{\text {th }}$ century for multiphase flow (Lokendra Pal, 2006). The permeability coefficient depends on both material and the fluid properties. The greater the $\mathrm{K}$ value is, the higher will the flow rate be.

Darcy's law is expressed by:

$$
Q=\frac{K \Delta P}{\eta \Delta L} A
$$

where, $\mathrm{Q}$ is the flow rate, $\mathrm{K}$ is the permeability coefficient, $\Delta \mathrm{P}=$ Pressure difference, $\mathrm{A}$ is the cross sectional area of the flow, $\eta$ is the fluid viscosity and $\Delta \mathrm{L}$ is the flow length.

Permeability is expressed in Darcy or millidarcys and most of the oil reservoirs are in the range of ten to several hundreds of millidarcys. Figure 2 shows the permeability of different types of commonly available rocks.

\footnotetext{
${ }^{1}$ OLGA-Roxs is a modelling software dedicated for oil field simulation. http://www.prod.software.slb.com ${ }^{2}$ http://www.tecplot.com
} 


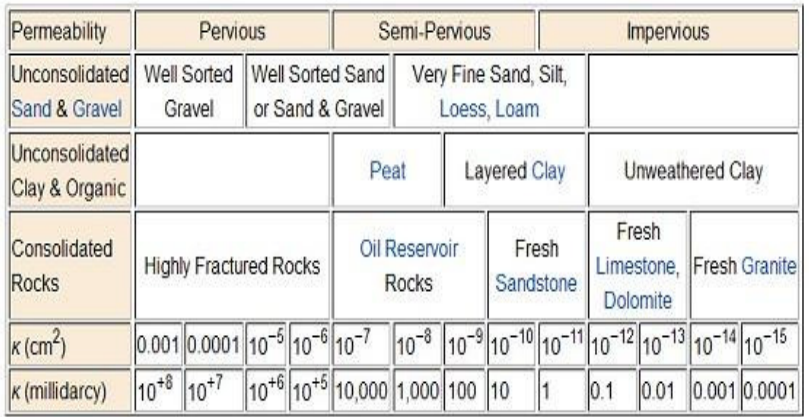

Source: modified from Bear, 1972

Figure 2. Permeability of common rocks (Logs).

\subsection{Relative Permeability}

The relative permeability is defined for multiphase flows. The effective permeability of a component of the multi-phase flow is given by the relative permeability. Darcy's law can be modified for a multiphase flow as expressed in Equation 3.

$$
Q_{i}=\frac{K_{i} \Delta P_{i}}{\eta_{i} \Delta L} A \quad i=1,2, \ldots \ldots, n
$$

The subscript $i$ indicates the parameters of $i$ th phase and $K_{i}$ is the of phase permeability of the component $i$. Several laboratory experiments have shown that the sum of the effective permeability is less than the absolute permeability (A. B. Zolotukhin, 1997).

$$
\sum_{i=1}^{n} K_{i}<K
$$

Relative permeability is the ratio between effective permeability of the respective phase and the absolute permeability as shown in Equation 4. Therefore, the sum of the relative permeability components must also be less than 1. Even though the effective permeability is a function of several parameters such as rock properties, fluid properties, absolute permeability fluid saturation and reservoir condition (pressure, temperature), the relative permeability depends on the fluid saturation and structure of the porous medium (A. B. Zolotukhin, 1997). However, the relative permeability can be assumed as a function of only saturation due to its strong correlation with saturation.

$$
K_{r i}=K_{i} / K
$$

Saturation of a particular fluid is denoted as the ratio of fluid volumes to pore volume as expressed in Equation 5. Though several attempts have been made to calculate the relative permeability on a theoretical basis, so far the commonly available data has been taken from experimental investigations. For two phase flows typical permeability curves are shown in Figure 3.

$$
\text { Saturation of Oil }\left(S_{o}\right)=\frac{\text { Volume of Oil }}{\text { Pore Volume }}
$$

One important remark on the curve is that the relative permeability becomes zero if the saturation of the corresponding phase is less than a specific value. The value is then said to be the residual saturation of the respective phase.

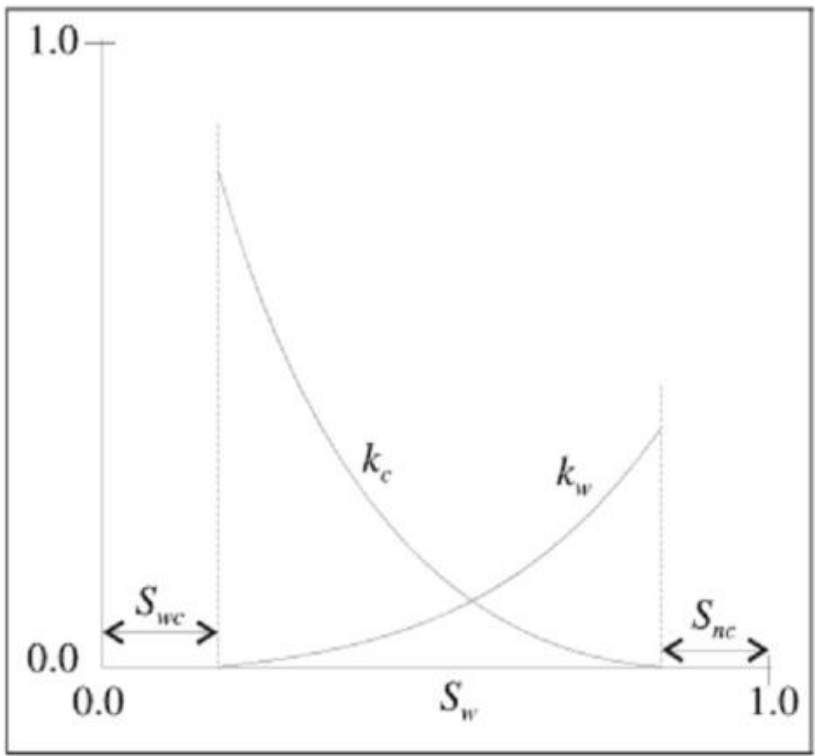

Figure 3. A typical oil-water relative permeability curve (A. B. Zolotukhin, 1997).

\section{Modelling and Simulation}

Models are built based on the properties found in the Grane field in the North Sea. Table 1. shows the reservoir properties of the Grane field. Even though, homogeneous reservoirs do not exist in real world, adequate parameters are used in simulation to evaluate real world challenges. A reservoir, having the length of $100 \mathrm{~m}$, the width of $201 \mathrm{~m}$ and the height of $30 \mathrm{~m}$ is considered. The geometry of the field is divided into a 3 -dimentional mesh with control volumes. The mesh is selected to obtain accurate results by using finer grids closer to well and coarser grids apart from the well. The considered reservoir with control volume distribution is shown in Figure 4. The horizontal well is placed along the $\mathrm{x}$-axis in the reservoir model. Reservoir properties such as permeability, porosity and relative permeability are included as input parameters to the model. Another two important inputs to the model are initial conditions and boundary conditions. Under the boundary conditions, the properties and the concentrations beyond reservoir margins are defined. If the aquifer is large enough, pressure at the lower boundary can be considered as a constant during the 
simulation. The well position in the reservoir block is also given as boundary conditions. The fluid concentration and saturation at initial time are given as initial conditions.

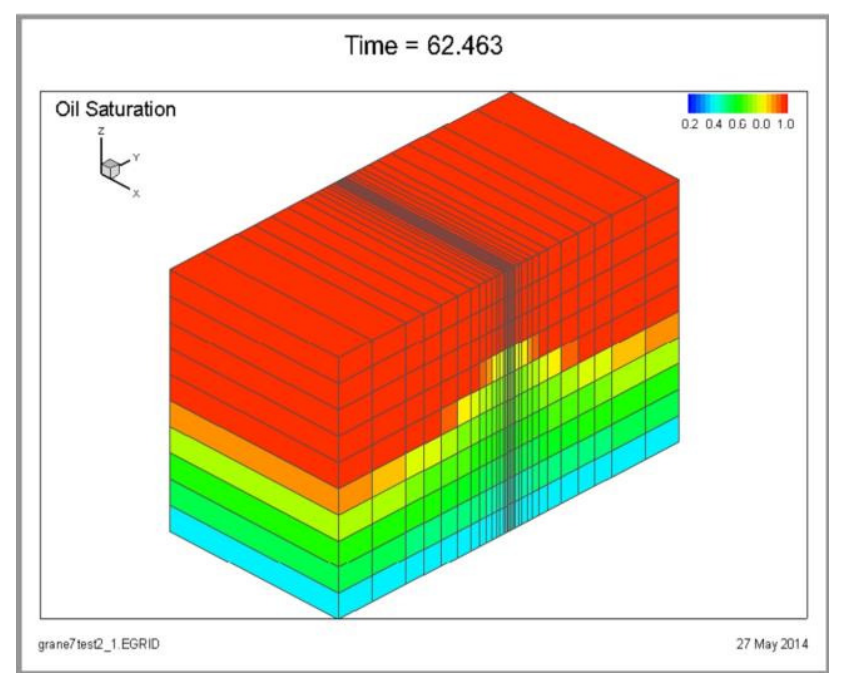

Figure 4. Control volume distribution.

Fluid flow variations along the horizontal well during normal operation can be inspected with the well model of interest. Simulations have been carried out with one Inflow Control Device (ICD) along the horizontal well, even though a real well has number of ICDs. The diameter of the ICD is $9 \mathrm{~mm}$. Based on the single-valve model, more complex models can be simulated in future with less effort. For the comparison, only a variable of interest is changed while keeping all other variables constant in order to avoid the impacts from them. Another attractive feature in the OLGA model is the availability to observe dynamic variations of each subcomponent during simulations. This feature is useful to identify errors and to introduce appropriate parameters of individual subcomponent. Trial and error method is used to introduce convenient parameters for the model.

Table 1. Reservoir properties in Grane field.

\begin{tabular}{|l|c|}
\hline \multicolumn{1}{|c|}{ Property } & Value \\
\hline Pressure (bar) & 176 \\
\hline Temperature (C) & 76 \\
\hline Porosity (\%) & 33 \\
\hline viscosity (cP) & 12 \\
\hline Thickness (m) & 31 \\
\hline Permeability (D) & 10 \\
\hline
\end{tabular}

In order to save the simulation time and the memory space are important in any kind of finite element methods. Simulations can be done with relatively large time steps to minimize the running time. Even though precise results are not given with large time steps, information taken from the simulations is useful to get general idea and to cut down errors prior to the final simulation. The selection of plotting variables is also decisive to reduce the running time.

This study is mainly focused on the influence of the relative permeability on flow rates, the water breakthrough time and the oil extraction efficiency. Figure 5. shows the relative permeability data found in literature and the graphs are created using polynomial regressions. Three different cases are concerned for the comparison where the residual water and the residual oil values are distinct from each other. At the beginning of well, the well surrounding is saturated with oil. During the simulation oil saturation throughout the reservoir is reduced, while the water saturation starts to increase gradually. However, water will not enter the wellbore until the water saturation around the well exceeds its own residual limit. Theoretically, the oil extraction can be continued till the saturation falls down to its residual limit. Residual oil in the reservoir remains in abandoned oil fields after operation is finished.

\section{Results and Discussions}

Three cases are simulated for 200 days and the comparison of the results are mainly focused on water breakthrough, liquid flow rates, accumulated liquid volumes and the reservoir oil saturation profiles. The results gave different values/profiles for each case so that the effect of the relative permeability is reflected. Figure 6. shows the accumulated total liquid volumes with respect to time. The average total liquid flow rates are represented by the gradients of respective graphs. The total flow rates prior to breakthrough are approximately $337 \mathrm{~m}^{3} /$ day. Gradients of the curves change with time. At the beginning, the flow rate is maintained at constant level until the breakthrough occurs and thereafter the total flow rate starts to fluctuate with time. Subsequently, the total flow rate and oil flow rate achieve different values due to the multiphase flow after the breakthrough.

The reason for the total flow rate deviation, from a constant value after the breakthrough, can be explained with the use of relative permeability and Darcy's equation for multiphase flow. The sum of relative permeability values is lower than absolute permeability. The total flow rate is then the sum of individual flow rates in the multiphase flow. Furthermore, the total flow rate depends on the relative permeability and the viscosities of each component as well. Theoretically, viscosity values would compensate the effect of relative permeability as shown in Equation 3 and might lead to a higher flow rates. 


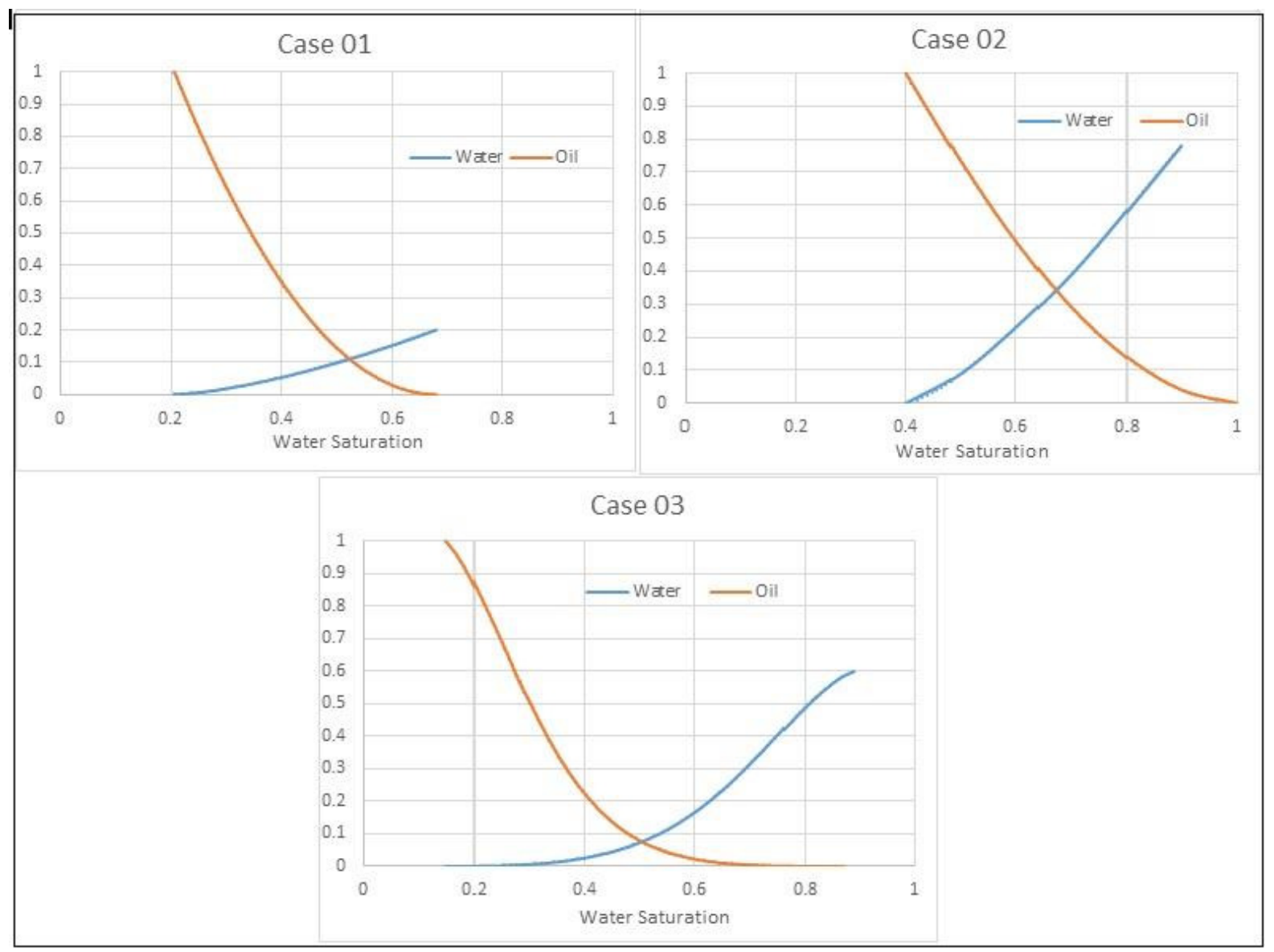

Figure 5. Relative permeability values in three cases.

However, total flow rate is always less than any single phase flow rate in practice. In this paper, oil-water multiphase flows are considered. The simulation results show that the total flow rates are decreased after the breakthrough. It seems that the relative permeability values of the multiphase flow influence in the flow rate reductions. The liquid flow rate is continuously changed after the breakthrough since the water saturation near the well is changed and then the relative permeability varies accordingly. Hence, the flow rate changes after the breakthrough can be well described with the dynamic variation of the relative permeability throughout the reservoir with time.

Table 3 shows the information about the water breakthrough for each case. The accumulated oil curves have the same characteristic since oil is the only component before the multiphase condition is achieved. The water saturation throughout the reservoir is an important factor to understand the variation of the breakthrough time. Multiphase phenomenon could be approached within the reservoir even before water enters to the well bore due to the water coning profile. It is important to note that water cone is formed above the aquifer at the beginning of the operation and the water saturation inside the cone should not necessarily be $100 \%$ saturated water. Table 3 shows the saturation at the grid $(1,16,4)$ where the well is located. Water saturations noticed at the breakthrough point are equal to the residual saturation for each case. Saturated values at any given time and any location can be picked up by using Tecplot data.

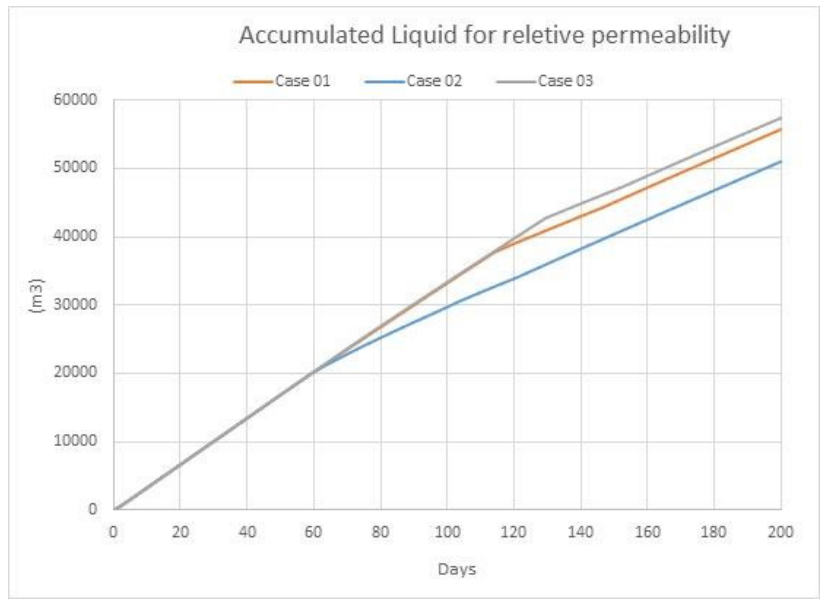

Figure 6. Accumulated Liquid profiles vs life time. 
Table 2. Liquid Volumes after 100 days.

\begin{tabular}{|c|c|c|}
\hline \multirow{2}{*}{ Case } & \multicolumn{2}{|c|}{ Volume $(\mathrm{m3})$} \\
\cline { 2 - 3 } & Liquid & Oil \\
\hline Case 01 & 33219 & 28672 \\
\hline Case 02 & 29717 & 25980 \\
\hline Case 03 & 33367 & 30973 \\
\hline
\end{tabular}

If water flows with the same speed within the reservoir until breakthrough happens, the water cone must have the same profile for every case. Figure 8 shows the oil saturation profile at the breakthrough time for the given cases. Further, the breakthrough time should be delayed according to the residual saturation so that the latest breakthrough time should occur, for Case 02, which has the highest residual water saturation. However, the latest breakthrough happens, in Case 03, which has the lowest residual water saturation. This deviation implies that the breakthrough is not only affected by the water saturation. This paradox can be explained using relative permeability graphs in Figure 5. Even though the water breakthrough happens at the residual saturation of water at the well-grid, water dispersion throughout the reservoir does not occur in the same rate due to the multiphase flow towards the well. When the oil saturation is closer to the residual level, the relative permeability of water increased exponentially (see Figure 3). When oil approaches its residual limit, the relative permeability of water is stabilized at absolute permeability and starts to act as a single phase flow. This nature of relative permeability leads to early breakthrough. The mobility of the water phase is highly activated at low oil saturations, especially around the residual limit and less activated for high oil saturations. Simulation results have given breakthrough points in the cases 01, 02, and 03 respectively. Therefore, it can be concluded that the influence of water relative permeability is affected than the residual saturation for the water breakthrough. The breakthrough time paradox is fully explained by using relative permeability curves and residual oil saturation of the multiphase within the reservoir before the breakthrough.

Accumulated oil volumes are shown in Figure 7 where the oil flow rates are gradually decreased after the water breakthrough. The reduction of oil flow rates after the breakthrough can be highlighted as a common observation in every case. However, the decreasing rates are different for each case. The highest decreasing rate is shown in case 02 and the lowest is shown in case 03. Total flow rate is also reduced after the breakthrough as discussed earlier. Case 01 and 03 have approximately similar dynamic behaviors in total flow rate curves. Therefore, it is easy to investigate and compare the oil flow behaviors of these two cases after the breakthrough.

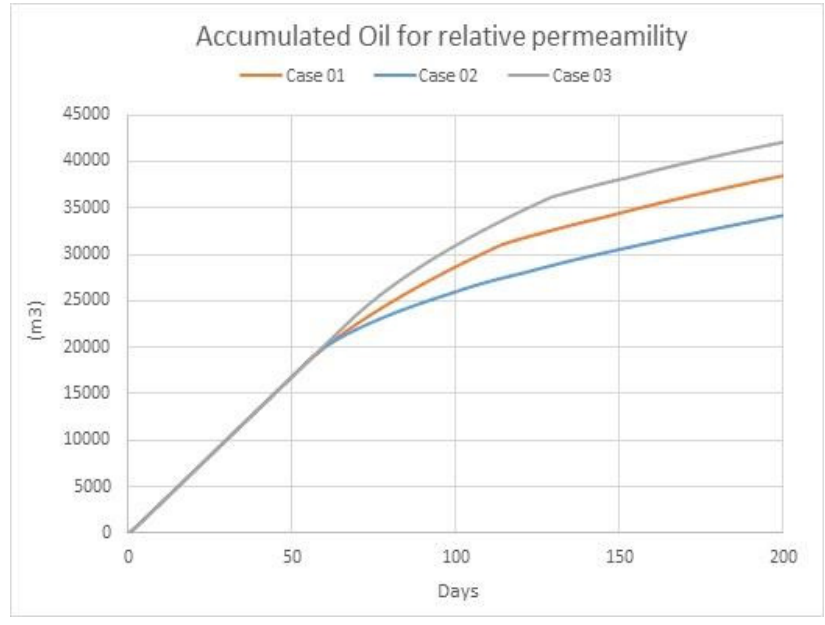

Figure 7. Accumulated Oil profile vs life time.

Gradients of the accumulated oil curves have almost a similar variation and curves are approximately parallel a long after the breakthrough. Accumulated volumes seem to converge to a certain value as shown in Figure 7 and this value is the highest for case 03. Therefore, case 01 produces less oil with the same reservoir size than case 03. Moreover, it can be concluded that the delayed water breakthrough will produce higher oil volume for the same total fluid volume. The oil recovery factor can be upgraded by delaying the water breakthrough.

Even though the residual oil saturation is zero, in case 02 , the lowest amount of oil is produced in this case. The observation reveals that lower oil residual reservoirs are not always producing higher oil volumes and the influence of the relative permeability is stronger than the oil residual value in some fields. Result inspections of the oil reservoir saturation profile shows that the narrowest water cone appeared at the earliest breakthrough case for a given well-age.

Table 3. Breakthrough data with different relative permeability.

\begin{tabular}{|c|c|c|c|}
\hline Case & $\begin{array}{c}\text { Water } \\
\text { Breakthrough }\end{array}$ & $\begin{array}{c}\text { Liquid } \\
\text { Volume at } \\
\text { breakthrough } \\
(\mathrm{m3})\end{array}$ & $\begin{array}{c}\text { Water } \\
\text { saturation } \\
\text { at well } \\
\text { Grid }\end{array}$ \\
\hline Case 01 & 53 & 18193 & 0.29 \\
\hline Case 02 & 55 & 18688 & 0.44 \\
\hline Case 03 & 68 & 23025 & 0.26 \\
\hline
\end{tabular}




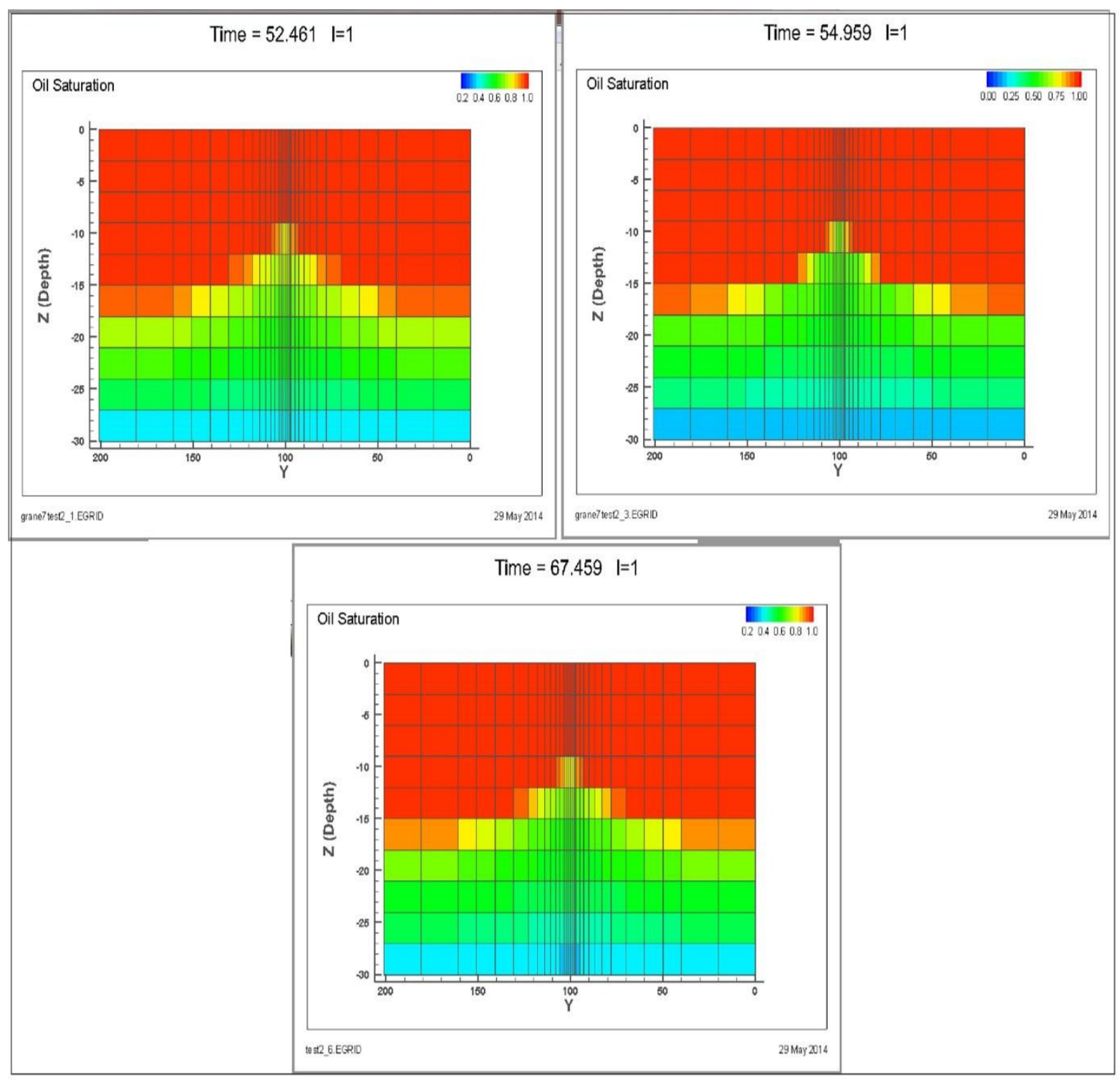

Figure 8. Oil saturation profile closer to breakthrough time (i) case 01 (ii) case 02 (iii) case 03.

\section{Conclusion}

This study was carried out to analyze the oil recovery characteristics in heavy oil reservoirs. A major challenge in oil fields is the decreasing oil production rate with time and the remaining amount of oil after well shutdown. Developing technologies have given some positive results on oil recovery factor. Water flooding and enhanced oil recovery methods are used successfully to increase the recovery. Water breakthrough is a strong challenge faced in heavy oil production and it causes increased water cut during the operation. Multiphase flow demands additional operational cost for separation. On the other hand, if water breakthrough is delayed, higher pure oil volume can be achieved. Therefore, delayed water breakthrough gives lower operational and maintenance costs.
Water breakthrough and multiphase flow strongly depend on relative permeability and the residual oil saturation in the reservoir. In this study, the influence of the relative permeability is investigated using different permeability curves and different residual oil saturations found in literature. The simulation results showed variations of water breakthrough time, accumulated total liquid, accumulated oil and the possible extracted amount of oil in a given reservoir with different permeability curves. Most of the observed behaviors can be explained by multiphase flow. The variation in water breakthrough times is a result of multiphase flow rate within the reservoir before the breakthrough. The influence of relative permeability is critical than the residual oil saturation on water breakthrough time. The results show that the case with minimum residual oil does not give the maximum recovery due to the flow characteristics in the reservoir. The mobility of water is significantly 
higher than of viscous oil, and the water can flow towards the well faster than oil from the bottom of the reservoir towards the well. This causes early breakthrough and water can enter to the wellbore even though there is a large amount of oil remaining in the reservoir. Enhanced oil recovery methods can be used to: manipulate the relative permeability, delaying the water breakthrough and obtain better production with high oil quality.

\section{References}

A. B. Zolotukhin, J. R. U. Introduction to reservoir engineering. Stavanger 1997.

Logs, E. Oil on my shoes. Disponível em: < http://www.geomore.com/porosity-and-permeability-2/ $>$. Acesso em: 2014/05/15.

Lokendra Pal, M. K. J., Paul D. Fleming. A Simple method for calculation of the permeability coefficient of porous media. TAPPI, 2006.

P. Zitha, R. F., D. Zornes, K. Brown, and K. Mohanty. Increasing Hydrocarbon Recovery Factors. Society of Petroleum Engineers 2011. Disponível em: < http://www.spe.org/industry/docs/recoveryfactors.pdf $>$. Acesso em: 2014/05/20.

Speight, J.; Speight, J. G. Enhanced recovery methods for heavy oil and tar sands. Houston, TX: Gulf Pub. Co. 2009. 